\title{
Limits on Achievable Intensity Reduction with an Optical
}

\section{Occulter}

\author{
Wasyl Wasylkiwskyj, ${ }^{1, *}$ Shahram Shiri ${ }^{2}$ \\ 'Electrical and Computer Engineering Department, The George Washington University, \\ Washington, D.C. 20037, USA \\ ${ }^{2}$ The Optics Branch, NASA/Goddard Space Flight Center, Greenbelt, MD 20771, USA \\ "Corresponding author: wasylkiw@gwu.edu
}

\begin{abstract}
We examine the degree to which the on-axis intensity of a normally incident electromagnetic wave within the visible spectrum can be reduced through shadowing by a suitably designed occulter. As is well known, blockage of light by a circular disk is partially negated by the formation of a bright spot at the geometrical center of the shadow region. The presence of this so called Poisson spot has been troublesome in astronomy. In particular, the NASA Terrestrial Planet Finder's mission, based on an external occulter, requires the suppression of starlight by many orders of magnitude. Two approaches have been proposed to deal with this issue. One approach is to use a partially transparent disk, the other an impenetrable body with a symmetric petal rim structure. We examine both approaches and examine their limitations. For a petal style occulter we find that the practical limit on the achievable intensity reduction is dictated by the necessarily finite radii of curvature of the petal tips. Our results show that the upper bound on the achievable intensity reduction is again about ten orders of magnitude. For the transparency we
\end{abstract}


derive an optimum circularly symmetric transparency function and obtain numerical results using the parameters appropriate to the NASA's Terrestrial Planet Finder mission. The results show that in practice to obtain an intensity reduction over the required spectral range, one would be able to use a partially apodized mask that would suffice the requirements as set by the mission.

OCIS codes: $350.1260,350.1270,350.6090$

\section{Introduction}

The Poisson Spot (also referred to as the spot of Arago) has been a controversial topic since its discovery in 1723 by Miraldi. Almost hundred years later in 1818 Poisson, a member of French Academy of Science and an advocate of the corpuscular theory of light, concluded that Fresnel's wave theory would predict a bright spot at the center of the shadow behind a circular obstruction. This prediction appeared to violate common sense and for Poisson served as evidence for the refutation of the wave theory of light. Later experiments by Arago verified the presence of this bright spot, and thereby played a significant role in validating the wave theory. Subsequently, interest in this phenomenon appears to have receded into the background and continued to be mentioned primarily in its historical context. More recently interest in the Poisson Spot has been revived in the astronomy community. The Terrestrial Planet Finder Missions funded by NASA is planning to perform direct imaging of exosolar planets to identify earth-like planets orbiting bright stars. Since starlight is many orders of magnitudes brighter than light reflected by an orbiting planet, its detection presents a scientific and technological challenge. Because of the intensity enhancement by the Poisson Spot., a conventional circular disk can not be used to reduce the starlight brightness. Thus an immediate task is the identification of techniques that 
effectively eliminate this spot. In the astronomy community, a number of researchers have addressed this problem by analyzing the effectiveness of a variety of transparency functions as apodizer masks. For instance, Copi and Starkman [1] modeled a square mask with a transmission function based on fourth-order Taylor series. They optimized the occulter using on-axis nulling and were able to obtain an intensity reduction of $4 \times 10^{-5}$. Nisenson \& Papaliolios [2] proposed a square crosss-apodization in the image plane. The intensity reduction achieved is very sensitive to the choice of the apodization function, and slight changes result in significant degradation in performance. Kuchner and Traub [3] analyzed masks with a sinc ${ }^{2}$ function as well as 8-th order polynomial for off-axis telescope systems. Similarly, Carson [4] analyzed and compared several models of graded occulting masks at the focal plane for NIR wavelengths. He investigated masks with transparencies using exponential, Gaussian, sinc, and 8-th order polynomial functions. The results showed that an exponential mask achieved the best intensity reduction, which turned out to be only about 2.3 magnitudes in the $2.0-4.0$ micron range.

In this paper, we investigate the limits on performance of two techniques: a circular disk with a partial transparency (apodization) function and a circularly symmetric solid occulter with a periodic boundary perturbation in form of "petals". The choice of the latter shape is motivated by the analyses of other investigators, in particular, Cash et. al. [5] and Vanderbei et.al. [6].

Even though occulter, to be of any use at all, must suppress the light intensity in the geometrical shadow region over a specified finite area surrounding the central axis, here we confine our analysis only to the central axis. One justification for this restriction is that meeting an intensity reduction requirement on the central axis is a necessary condition for the effectiveness of any occultation approach; the other is a relative simplicity of the analysis and modest numerical 
effort. In any case, the extension of our formulation to encompass the entire geometrical shadow zone is relatively straight forward and we shall report on it in a follow up paper.

Even though our present analytical formulation is quite general, the numerical results focus primarily on the parameters of the Terrestrial Planet Finder Mission, shown schematically in Figure 1, Planet

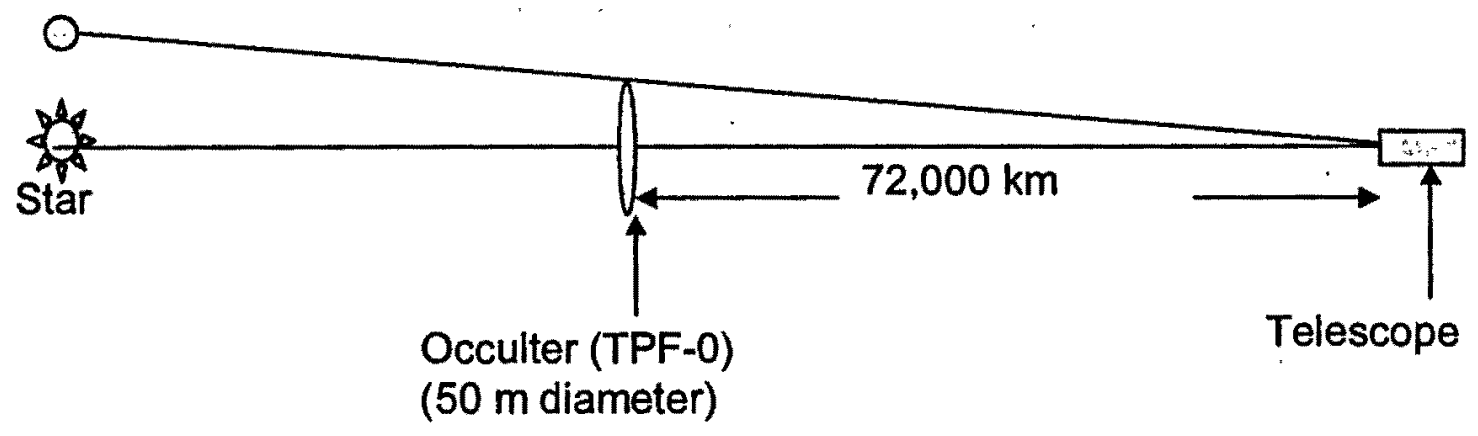

\footnotetext{
Required Intensity Reduction of Direct Starlight at the Telescope location: Spectral Range: 0.4 - 1.1 mlcrons Corresponding Fresnel number Range: $0.0461-0.1267$
}

Figure 1. Space-based optical occulter for detection of earth-like planets

In section 2 we formulate the circularly symmetric petal style occulter. Since the exact functional form of the petal pattern used by Vanderbei was not readily available, we use an analytical form that appears to closely mimic his geometrical pattern. In any case, the intensity reduction achieved does not appear to be overly sensitive to the functional form of the petals. Also, the number of petals also appears to play only a minor role (for our numerical results we use 6 petals). What does matter is the radius of curvature at the petal tips. Indeed, our functional petal form includes the radius of curvature as a parameter. We find that as the petal tip radius is 
decreased the intensity suppression continues to improve. In fact if the tip radius of curvature is sufficiently small, very high suppression levels can be attained. How small can the tip radius of curvature be made? First, to be compatible with the postulates of Physical Optics (on which all our analysis is based), it must be much larger than the fee space wavelength. Second, it can not be so small that it can not be maintained in the space environment (the outer radius of our occulter is 25 meters!) For example, for a radius of curvature of $0.2 \mathrm{~mm}$ (about 500 wavelength at 0.4 microns) we get an intensity reduction of 7 orders of magnitude. To reduce the intensity by 10 orders of magnitude as reported in Cash et.al [5] and Vanderbei et.al. [6] we would have to reduce the radius of curvature to the absurd value of 0.3 microns. Even the $0.2 \mathrm{~mm}$ would probably not be practical. Unfortunately at more reasonable values, say $1 \mathrm{~cm}$, the suppression is only about 5 orders of magnitude.

Section 3 is dedicated to the intensity reduction problem for a circular disk with an arbitrary circularly symmetric transparency function. Using a polynomial representation we determine the coefficients that minimize the intensity over a specified segment of the central axis in the geometrical shadow region. We find that for this optimized transparency function the intensity suppression continues to improve as the order of the polynomial is increased. However once the order of the polynomial is increased beyond a certain maximum the improvement ceases. This saturation effect is governed by the limit on the achievable computational accuracy. In practice, this phenomenon would lead to extreme tolerance requirements on the transparency function. We quantify these requirements with the aid of the Singular Value Decomposition of the pertinent matrices. We find that for the parameters of interest to the Terrestrial Planet Finder Mission the limit on the intensity reduction is about 10 orders of magnitude. 


\section{Diffraction by Petal-Style Occulter}

The geometry of the problem for petaled-style occulter is shown in Figure 2 where the disk is opaque. The number of petals on the disk and the radius of curvature are the critical factor on determining the level of suppression obtained from the disk.

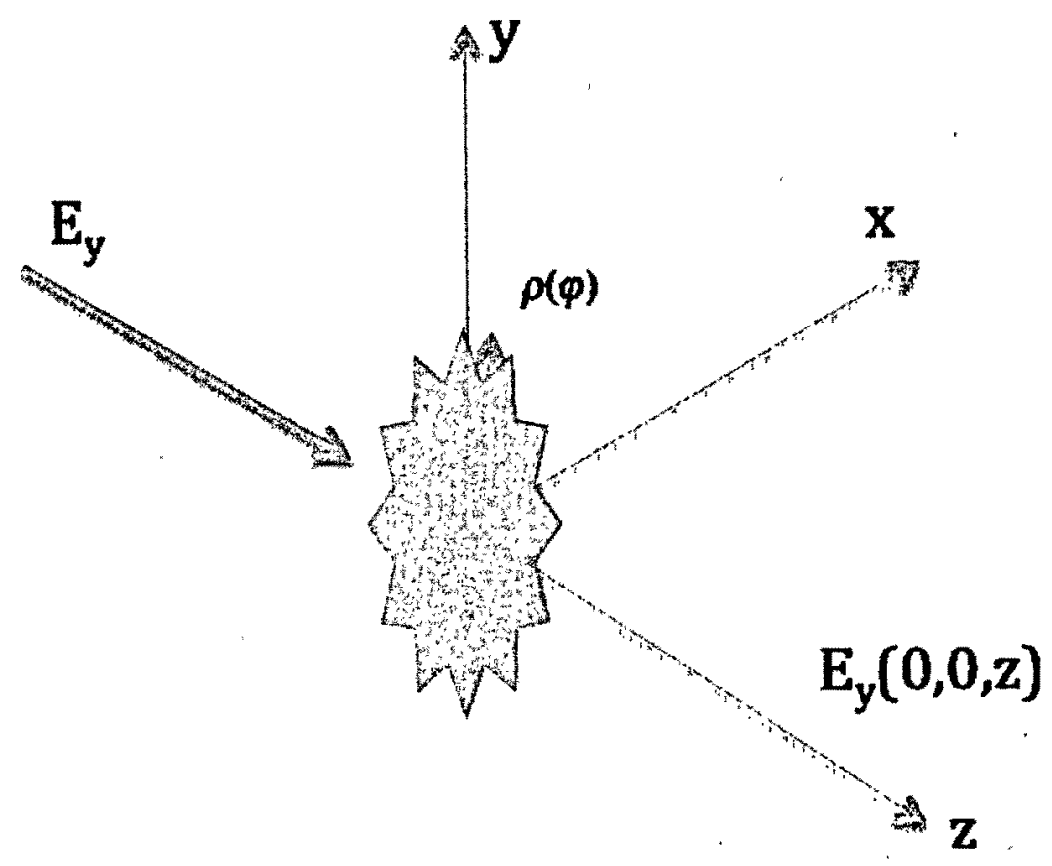

Figure 2. Geometry of petaled-style opaque occulter

The electric field along the central axis of the petaled occulter is derived from

$$
E_{y}(0,0, z)=\frac{A z}{2 \pi} \int_{0}^{2 \pi} \frac{e^{i k_{0} \sqrt{z^{2}+\rho^{2}(\varphi)}}}{\sqrt{z^{2}+\rho^{2}(\varphi)}} d \varphi
$$

Where $A$ is the amplitude of the occulter transparency, $\rho(\varphi)$ is the radius on the surface of the occulter, and $z$ is the distance behind the occulter. For a circular disk $\rho(\varphi)=R$, independent of $\varphi$, we can obtain the field at the center of the Poisson Spot from 


$$
E_{y}(0, z)=A z \frac{e^{i k_{0} \sqrt{z^{2}+R^{2}}}}{\sqrt{z^{2}+R^{2}}}
$$

The equation (2) is the general electric field on the center of the circular disk where $R=\max [\rho(\varphi)]$ and $0 \leq \varphi \leq 2 \pi$. The distance in this formulation is normalized to the reciprocal of the Fresnel number at any point $z$ by $r_{f}=\frac{2 \pi z}{R^{2} k_{0}}=\frac{z}{\left(R^{2} / \lambda\right)}=\frac{1}{F(z)}$

The relative intensity on the occulter axis expressed in dimensionless form would be

$$
I\left(r_{f}, R / \lambda\right)=\left|\frac{E_{y}(0,0, z)}{A}\right|^{2}=\left(\frac{r_{f}}{2 \pi}\right)^{2}\left|\int_{0}^{2 \pi} d \varphi \frac{e^{i 2 \pi(R / \lambda)^{2} \sqrt{r_{f}^{2}+(\lambda / R)^{2}[\rho(\varphi) R]^{2}}}}{\sqrt{r_{f}^{2}+(\lambda / R)^{2}[\rho(\varphi) / R]^{2}}}\right|^{2}
$$

\section{Fresnel Approximation of Petaled-Style Occulter}

The astronomy application of petaled-based occulter implies the radius of the symmetric occulter is considerably smaller than the distance between the occulter and the telescope, $\rho=z$, such that $F(z)=R^{2} / \lambda z=R / \lambda$. As a consequence one could use the Fresnel approximation to derive the electric field behind the occulter from

$$
E_{y}(0,0, z)=\frac{A z}{2 \pi} \int_{0}^{2 \pi} \frac{e^{i k_{0} \sqrt{z^{2}+\rho^{2}(\varphi)}}}{\sqrt{z^{2}+\rho^{2}(\varphi)}} d \varphi \cong \frac{A e^{\alpha_{0} \pi}}{2 \pi} \int_{0}^{2 \pi} e^{i k_{0} \rho^{2}(\varphi) / 2 z} d \varphi
$$

This derivation leads to the following simplification form of the normalized on-axis relative intensity,

$$
I\left(r_{f}\right)=\frac{1}{(2 \pi)^{2}}\left|\int_{0}^{2 \pi} e^{i \pi[\rho(\varphi) R]^{2} / r_{f}} d \varphi\right|^{2}
$$




\section{Intensity Suppression using a Transparency Function}

The geometry of the problem is shown in Error! Reference source not found. We are interested in intensity contrast ratio behind the disk. In this model, a normally planar wave polarized in y-direction is incident on a circular disk of radius $R$ with a circularly symmetric transparency (apodization) function $T(\rho)$, such that $T(\rho)=0$ corresponds to perfect opaqueness and $T(\rho)=1$ corresponds to perfect transparency. We choose the constraints $T(0)=0$, $T(R)=1$, and $\frac{d T(\rho)}{d \rho} \geq 0$

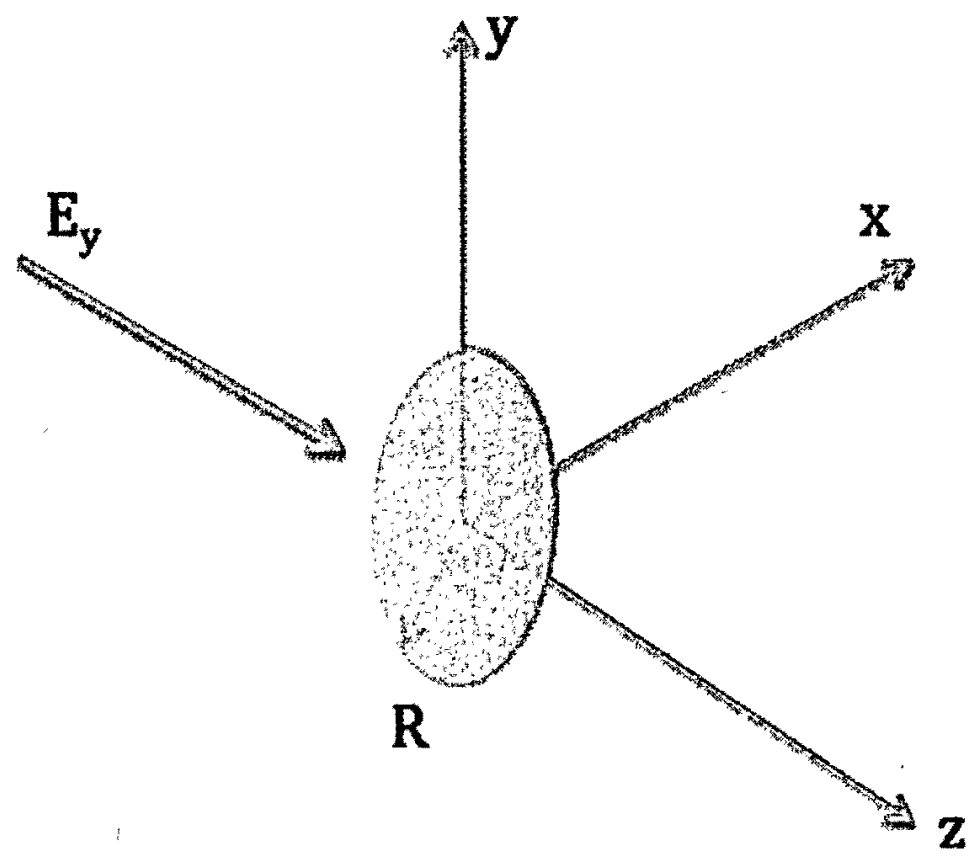

Figure 3, Geometry of occulter problem. Planar wave is incident into a circular disk with transparency function $T$

For such a disk the electric field on the axis of the geometrical shadow zone is given by (6),

$$
E_{y}(0, z)=A \int_{0}^{R} z \frac{e^{i k_{0} \sqrt{z^{2}+\rho^{2}}}}{\sqrt{z^{2}+\rho^{2}}} \frac{d T(\rho)}{d \rho} d \rho
$$


where $A$ is amplitude of the incident plane wave, $k_{0}$ is the free space wave number and $k_{0} R \gg 1$. In the special case of a perfectly opaque disk $T(\rho)=0, \quad 0 \leq \rho \leq R$ and $T(\rho)=1, \quad R<\rho<\infty$. In that case the derivative in (6) approaches a Dirac delta function at $\rho=R$ and we obtain

$$
E_{y}(0, z)=\frac{A z e^{i k_{0} \sqrt{z^{2}+R^{2}}}}{\sqrt{z^{2}+R^{2}}}
$$

which is the Poisson Spot intensity along the central axis. Upon introducing the dimensionless parameters $r_{f}=\lambda z / R^{2}$ (reciprocal of the Fresnel number) and $\hat{\rho}=\rho / R,(1)$ takes on the form

$$
E_{y}\left(r_{f}\right)=A r_{f}(R / \lambda) \int_{0}^{1} \frac{e^{i 2 \pi(R / \lambda) \sqrt{r_{f}^{2}(R / \lambda)^{2}+\ddot{\beta}^{2}}}}{\sqrt{r_{f}^{2}(R / \lambda)^{2}+\ddot{\beta}^{2}}} \frac{d T(\ddot{\rho})}{d \ddot{\beta}} d \ddot{\beta}
$$

For $z \square R$ (which is the only range of interest) (8) may be represented by the Fresnel approximation formula

$$
E_{y}\left(r_{f}\right)=A e^{i k_{0} z} \int_{0}^{1} e^{i \pi \vec{p}^{2} / r_{f}} \frac{d T(\ddot{\rho})}{d \ddot{\rho}} d \ddot{\rho}
$$

and the relative intensity along the optical axis is obtained from

$$
I\left(r_{f}\right)=\left|\int_{0}^{1} e^{i \pi \vec{p}^{2} / r_{f}} \frac{d T(\ddot{\rho})}{d \ddot{\rho}} d \ddot{\rho}\right|^{2}
$$

It is important to note that the relative intensity does not depend explicitly on the radius of the disk or the distance between the disk and the observation point, but only on the reciprocal of the Fresnel Number at the observation point.

We shall represent the transparency function in (10) by the M-th order polynomial

$$
T(\hat{\rho})=\sum_{\ell=1}^{M} c_{\ell} \hat{\rho}^{\ell}
$$


and determine the coefficients $c_{\ell}$ that minimize the arithmetic average of the relative intensity over a set of points within a specified segment of the central axis. Substituting (11) in (10) the relative intensity at any point $r_{f}(m), \quad m=1,2,3, \ldots, N$ is

$$
I\left[r_{f}(m)\right]=\left|\int_{0}^{1} e^{i \pi \hat{\rho}^{2} / r_{f}(m)} d \hat{\rho} \sum_{\ell=1}^{M} \ell c_{\ell} \hat{\rho}^{\ell-1}\right|^{2}=\sum_{\ell=1}^{M} \psi_{t m}
$$

where $\psi_{\ell m}=\ell \int_{0}^{1} e^{i \pi \hat{\rho}^{2} / r_{f}(m)} \hat{\rho}^{\ell-1} d \hat{\rho}$. The intensity $I_{a w N}$, averaged over $N$ positions, takes on the form

$$
I_{a v N}=\frac{1}{N} \sum_{m=1}^{N} I\left[r_{f}(m)\right]=\frac{1}{N} \sum_{m=1}^{N}\left|\sum_{i=1}^{M} \psi_{1 m} c_{1}\right|^{2}=\frac{1}{N} \sum_{i=1}^{M} \sum_{k=1}^{M} c_{1} c_{k}\left(\sum_{m=1}^{N} \psi_{1 m} \psi_{k m}^{*}\right)
$$

As a convenient short hand notation we define the matrix $A_{4 k}=\sum_{m=1}^{N} \psi_{1 m} \psi_{k m}^{*}$ and rewrite (13) in the following form:

$$
I_{a v N}=\frac{1}{N} \sum_{\ell=1}^{M} \sum_{k=1}^{M} c_{\ell} c_{k} A_{i k}=\frac{1}{N} \mathbf{c}^{T} \mathrm{Ac} \geq 0
$$

where

$$
\mathbf{c}=\left[c_{1} c_{2} c_{3} \ldots c_{M}\right]^{T}
$$

For complete transparency at the rim of the disk, (6) requires $\sum_{\ell=1}^{M} c_{\ell}=1$, or, equivalently $\mathbf{c}^{T} 1=1$ where 1 is a column matrix of ones. Using this as a constraint in the minimization of $\mathbf{c}^{T}$ Ac we take the variation with respect to $\mathrm{c}$ and set it to zero. Thus

$$
\delta\left[\mathbf{c}^{T} \mathrm{Ac}+q\left(1-\mathbf{c}^{T} \mathbf{1}\right)\right]=0 .
$$


where $q$ is the Lagrange multiplier. Since $\delta$ c is arbitrary, its multiplier may be set to zero yields and one finds

$$
\mathbf{c}=q\left(\mathbf{A}+\mathbf{A}^{T}\right)^{-1} \mathbf{1}
$$

Using the constraint $\mathbf{c}^{T} 1=1$, we obtain for the Lagrange multiplier

$$
q=\frac{1}{\mathbf{1}^{T}\left(\mathbf{A}+\mathbf{A}^{T}\right)^{-1} \mathbf{1}}
$$

so that the coefficients of the polynomial that minimizes the arithmetic average of (14) over $\mathrm{N}$ observation points are represented by the vector

$$
c_{o p t}=\frac{\left(A+A^{T}\right)^{-1} 1}{1^{T}\left(A+A^{T}\right)^{-1} 1}
$$

The preceding optimization ensures complete opacity only at the disk center. A more general form of the transparency function would exhibit complete opacity over a finite circular with radius $a$,i.e.,

$$
T(\hat{\rho})=\left\{\begin{array}{c}
0 ; 0 \leq \hat{\rho} \leq a / R \\
\sum_{\ell=0}^{N} c_{\ell} \hat{\rho}^{\ell} ; a / R \leq \hat{\rho} \leq 1
\end{array}\right.
$$

The coefficients $c_{\ell}$ in on (20) that minimize within the interval $a / R \leq \hat{\rho} \leq 1$ may be computed using (19) provided we replace $\hat{\rho}$ in (20) by $\frac{\hat{\rho}-a / R}{1-a / R}$

The expression for the intensity (12) must now be replaced by

$$
I\left[r_{f}(m)\right]=\left|\int_{a / R}^{1} e^{i \hbar \hat{\phi}^{2} / r_{f}(m)} d \hat{\rho} \sum_{\ell=1}^{M} \ell c_{\ell}\left(\frac{\hat{\rho}-a / r}{1-a / R}\right)^{\ell-1}\right|^{2}=\sum_{\ell=1}^{M} \psi_{l m}
$$

and 


$$
\psi_{\ell m}=\ell \int_{a / R}^{1} e^{i \pi \hat{\rho}^{2} / r_{f}(m)}\left(\frac{\hat{\rho}-a / R}{1-a / R}\right)^{\ell-1} d \hat{\rho}
$$

\section{Petaled-Style Occulter Model Results}

The occulter geometry was modeled as petaled-style disk to suppress the Poisson Spot along the central axis of normally incident plane waves. In this simulation, we used two functional forms of the occulter petals. In the first type, the radii of the curvature at the tips of the petals was calculated from

$$
r_{c}=R \frac{m\left(\frac{\pi}{N}\right) p \ln \left(\frac{1+p}{p}\right)}{2\left(1-r_{0}\right)+m\left(\frac{\pi}{N}\right) p \ln \left(\frac{1+p}{p}\right)}
$$

Where $p$ is the petal scaling factor usually unity, $N$ is the number of the petals, $m$ is the characteristic decay exponent that controls the petal shape, and $R$ is the occulter radius.

The second type of functional form of the occulter, the radius of curvature is obtained from,

$$
r_{c}=R \frac{\left(f_{\max }^{(2)}-f_{\min }^{(2)}\right) m\left(\frac{\pi}{N}\right)^{2} p}{\left(1-r_{\min }\right)\left[f_{\max }^{(2)}\right]^{(1-m)}+\left(f_{\max }^{(2)}-f_{\min }^{(2)}\right) m\left(\frac{\pi}{N}\right)^{2} p}
$$

Where $f_{\max }^{(2)}$ and $f_{\min }^{(2)}$ are obtained from $f_{\max }^{(2)}=\left\{\ln \left[\frac{1+p}{\sqrt{p}}\right]\right\}^{1 / m}$ and $f_{\min }^{(2)}=[\ln \sqrt{1+p}]^{1 / m}$. The simulations using first type petaled occulter is depicted in Figure 4. 


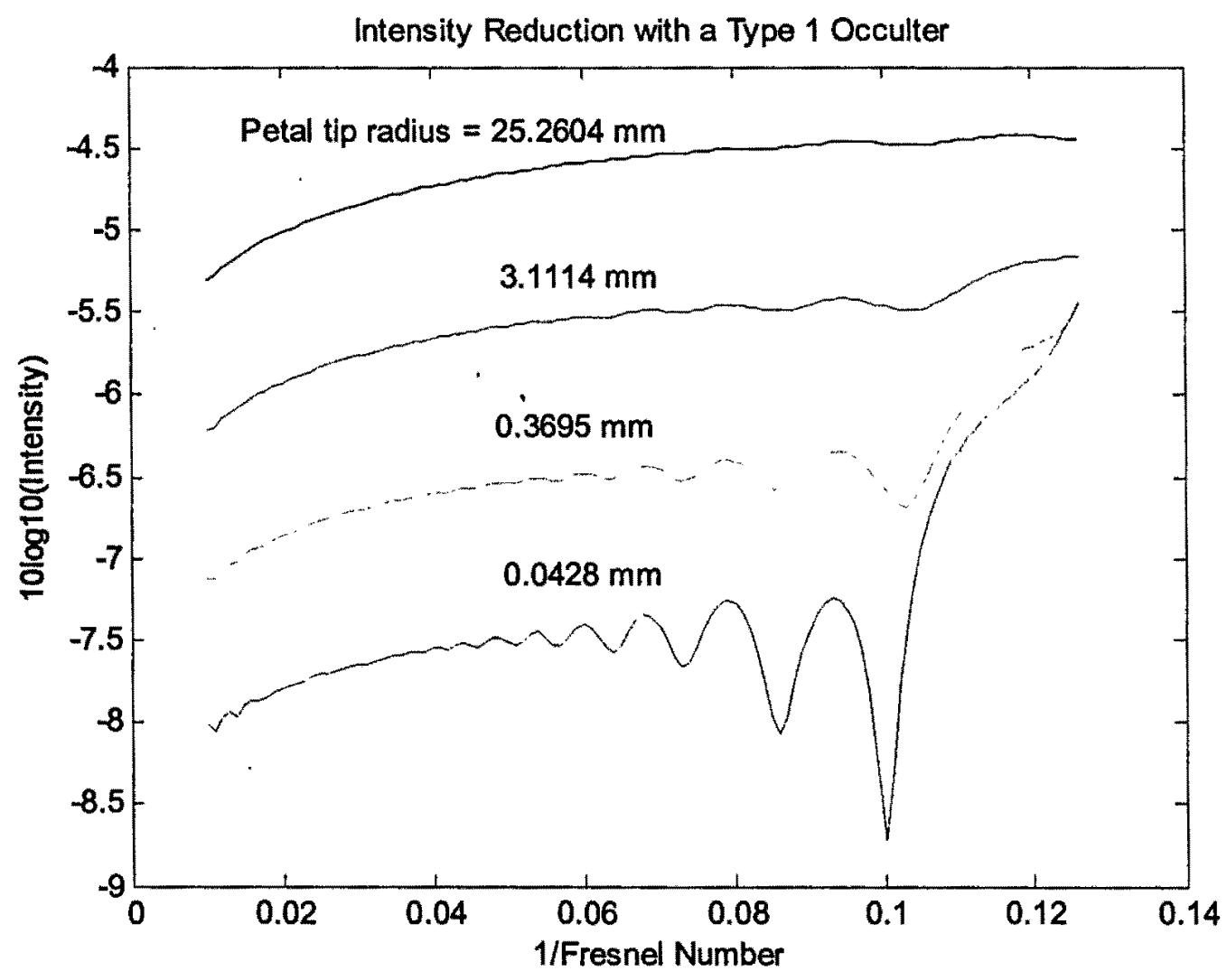

Figure 4, Intensity reduction using first type occulter

In this figure, the intensity reduction is normalized over the inverse of the Fresnel number for occulter with radius of 25 meters. The maximum reduction occurs as the radius of the petal tip decreases to 0.0428 millimeters. Similarly, the simulation of intensity reduction using second type occulter is depicted in Figure 5. The intensity reduction in this type of occulter generally follows the same as pattern as the type 1 occulter except the reduction occurs at smaller petal tips indicating a smoother curvature. 


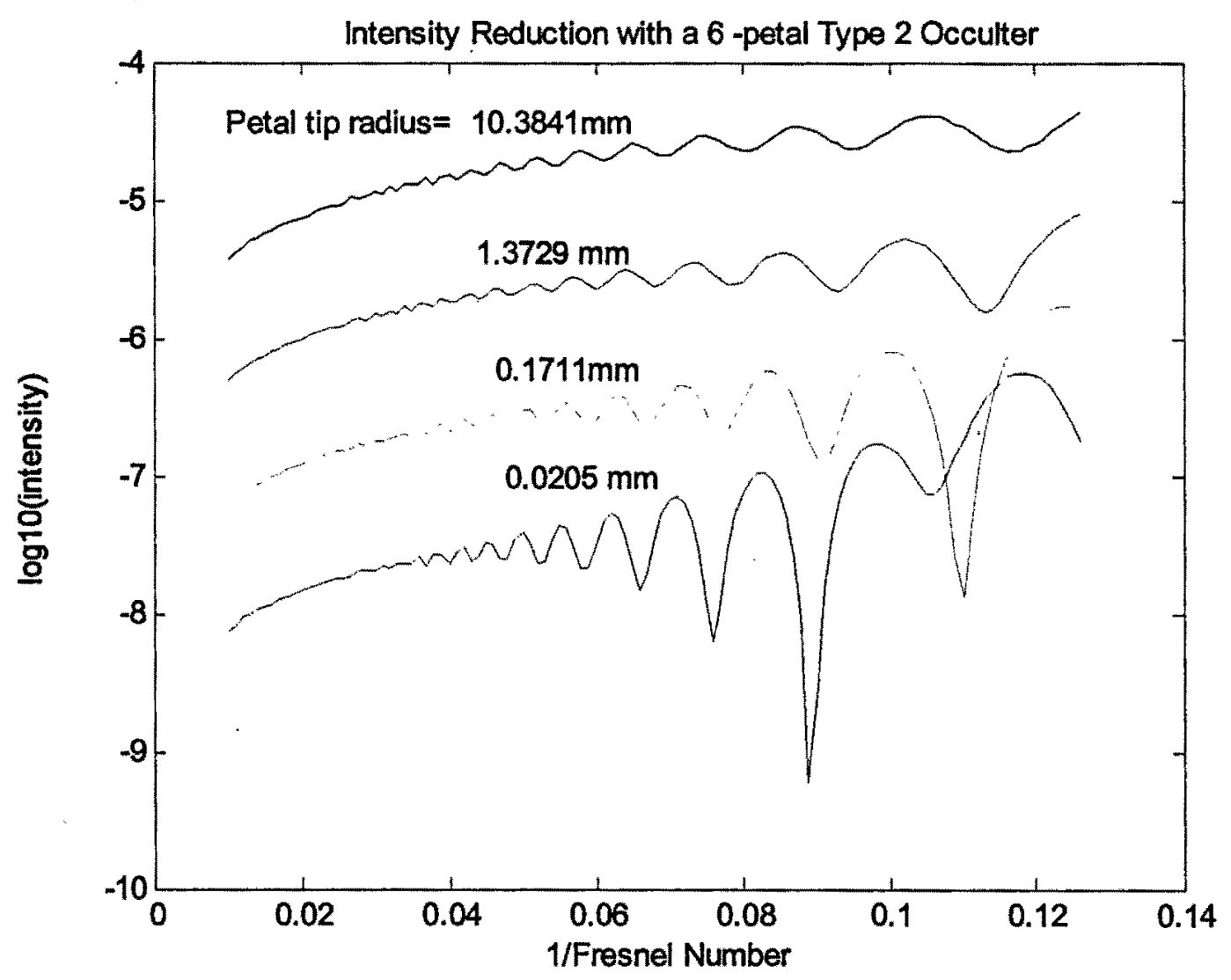

Figure 5, Intensity reduction using type 2 occulter petal tips

Figure 6 shows the intensity reduction of type 1 occulter could be significantly lower if the petal tips reduced to 0.544 microns. This reduction meets the requirements of missions such as direct imaging of Earth-like planet detections. 


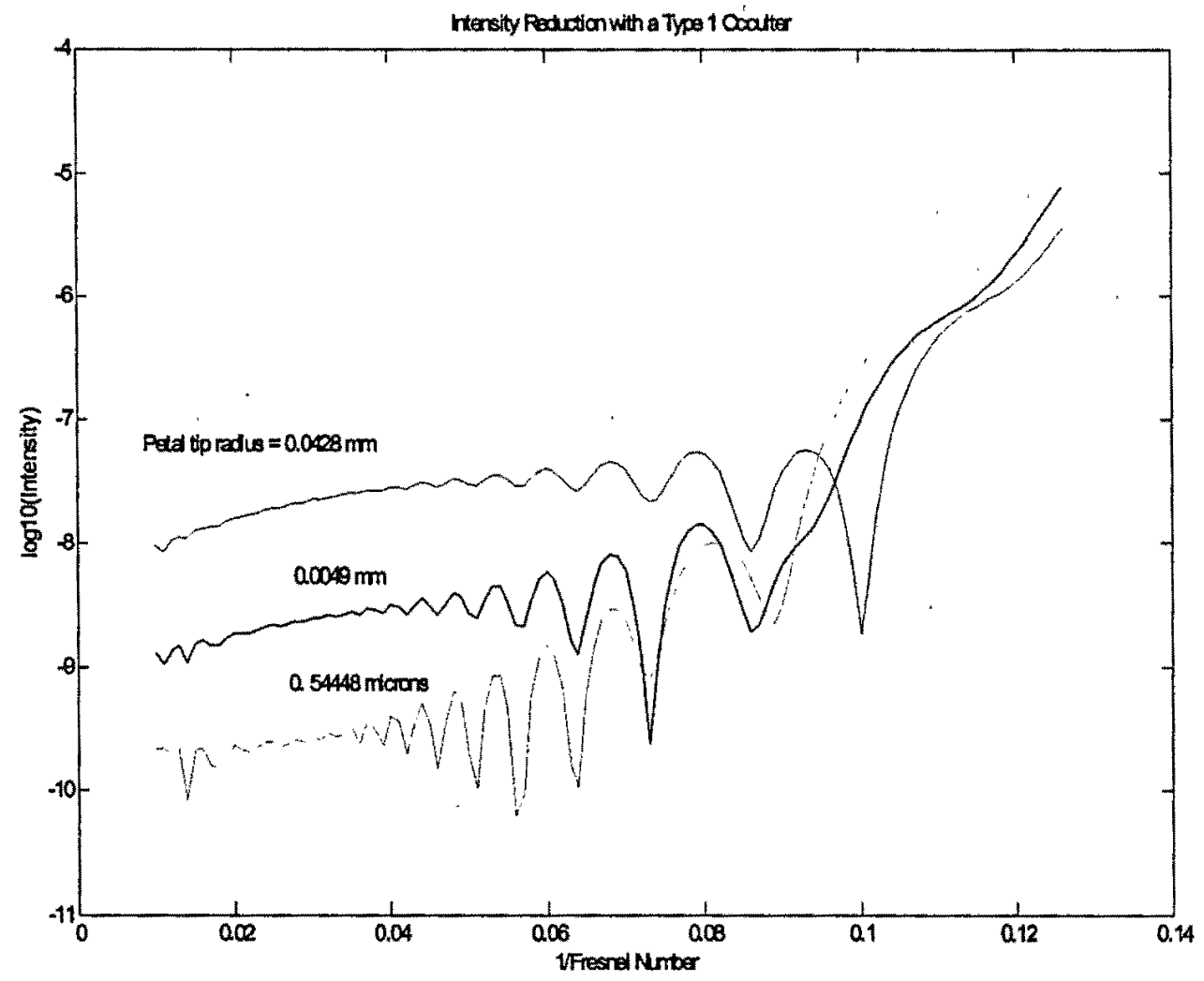

Figure 6. Maximum intensity reduction using type 1 occulter petal

\section{Transparent Circular Disk Model Results}

The circular occulter geometry subject to the transparency function, $T(\ddot{\rho})$, and constraint $\sum_{i=1}^{M} c_{i}=1$ was approximated with polynomials of $4^{\text {th }}, 8^{\text {th }}$, and $10^{\text {th }}$ order. Figure 7 depicts the comparison of the transparency function normalized to the occulter radius. A close inspection of this plot shows transparency function, S-shape, remains close to zero for almost half of the radius 
of occulter with an exponential increase on the transparency with solid plateau at the end.

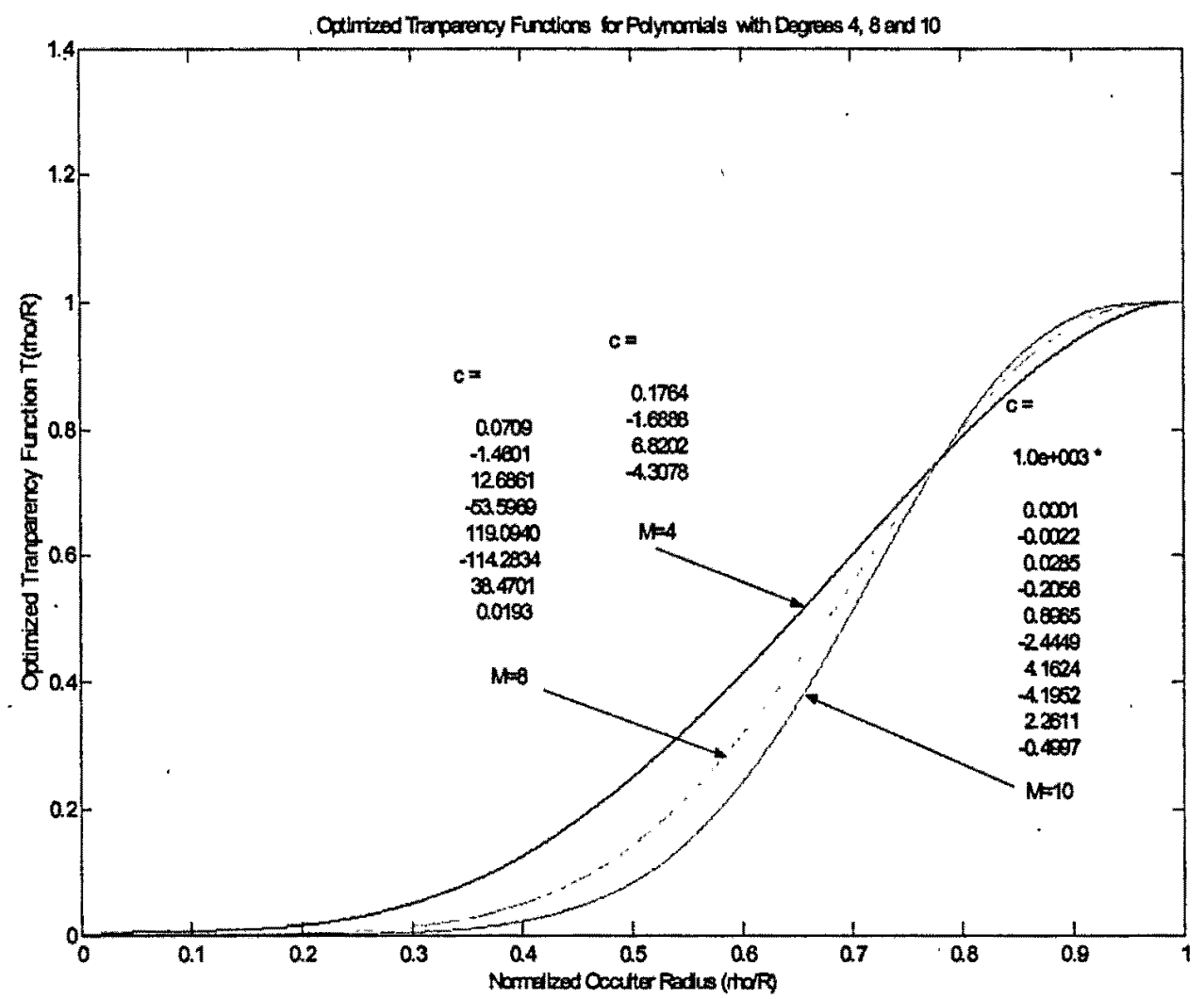

Figure 7. Comparison of optimized transparency function for polynomlals of 4 th, 8 th, and 10 th order.

In addition to above, we scaled the transparency function in abscissa such that the transparency is scaled away from center of the disk, to the right. Figure 8 shows scaled transparency functions for polynomials of $4^{\text {th }}, 10^{\text {th }}$, and $30^{\text {th }}$ order such that the start of the transparency begins at 0.29 of a normalized disk radius. The intensity reduction obtained from these scaled transparency functions are depicted in. The application of the transparency function in astronomy where the relative intensity along the optical axis needs to be reduced by several orders of magnitude in order to image the Exo-planets require large circular masks in orders of meters positioned in the space many thousand kilometers away from telescope. 


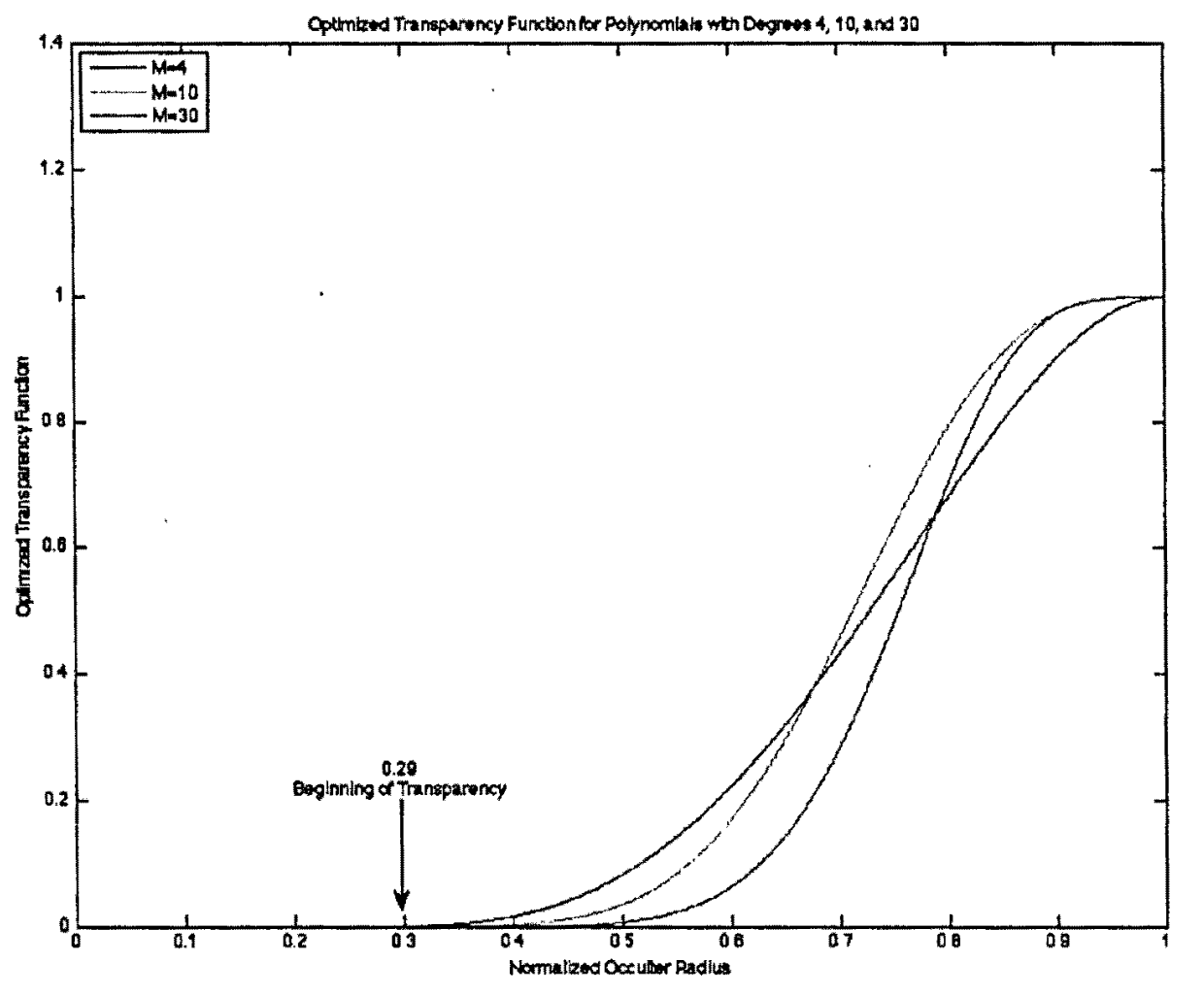

Figure 8. Comparison of optimized transparency function for polynomials of 4 th, 10th, and 30th order. 
As the results show, polynomial of $40^{\text {th }}$ order gives an average of 11 order of magnitude intensity reduction in the desired visible range of 0.4 microns to 0.8 microns.

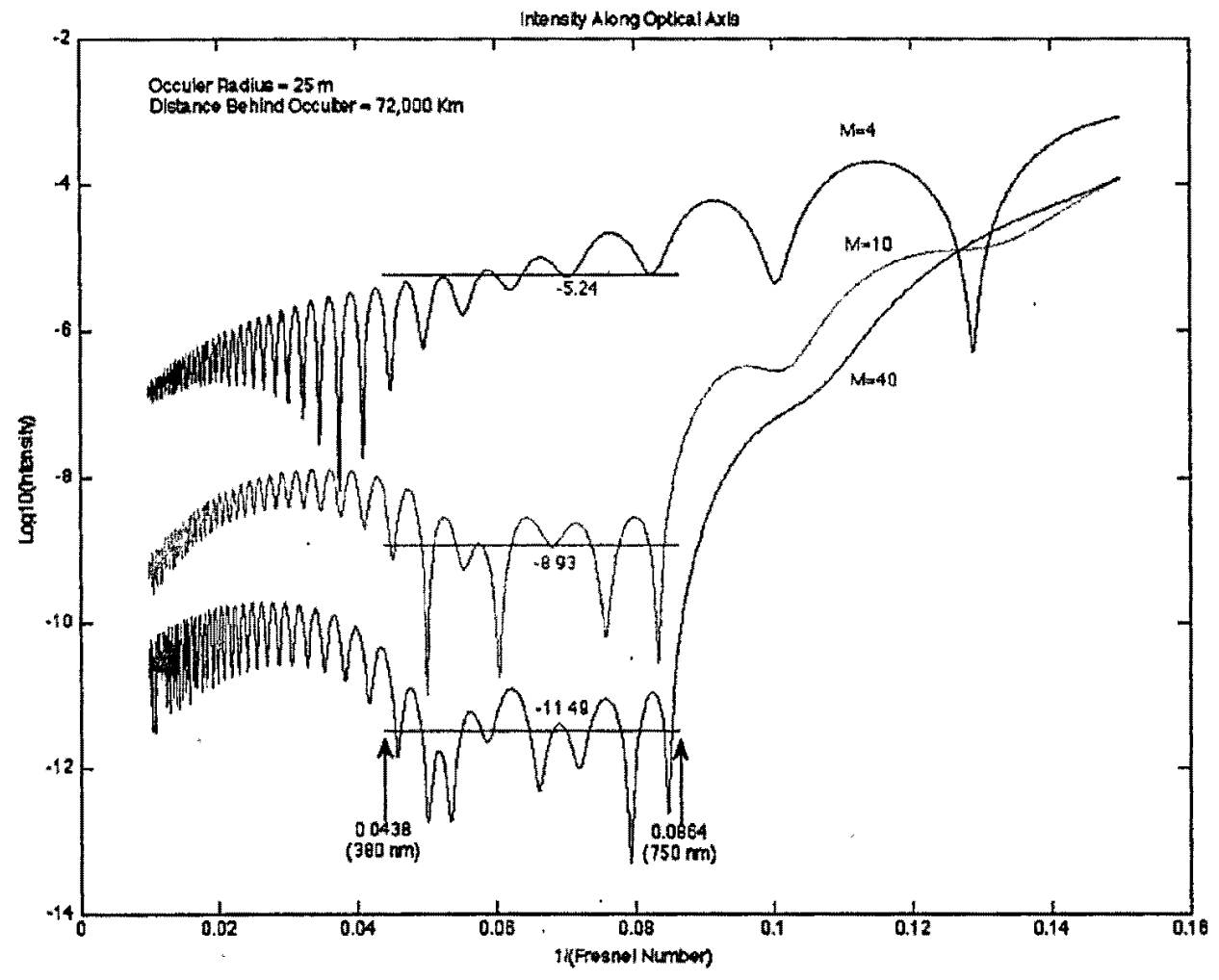

Figure 9. Intensity reduction along optical axis for scaled apodized circular disk.

\section{Sensitivity of Transparency Function}

The sensitivity of transparency function can be modeled by the perturbation of the coefficients, $\mathbf{c}_{\text {opt }}$, used in the polynomial function. The resulting normalized intensity reduction is shown in Figure 10. 


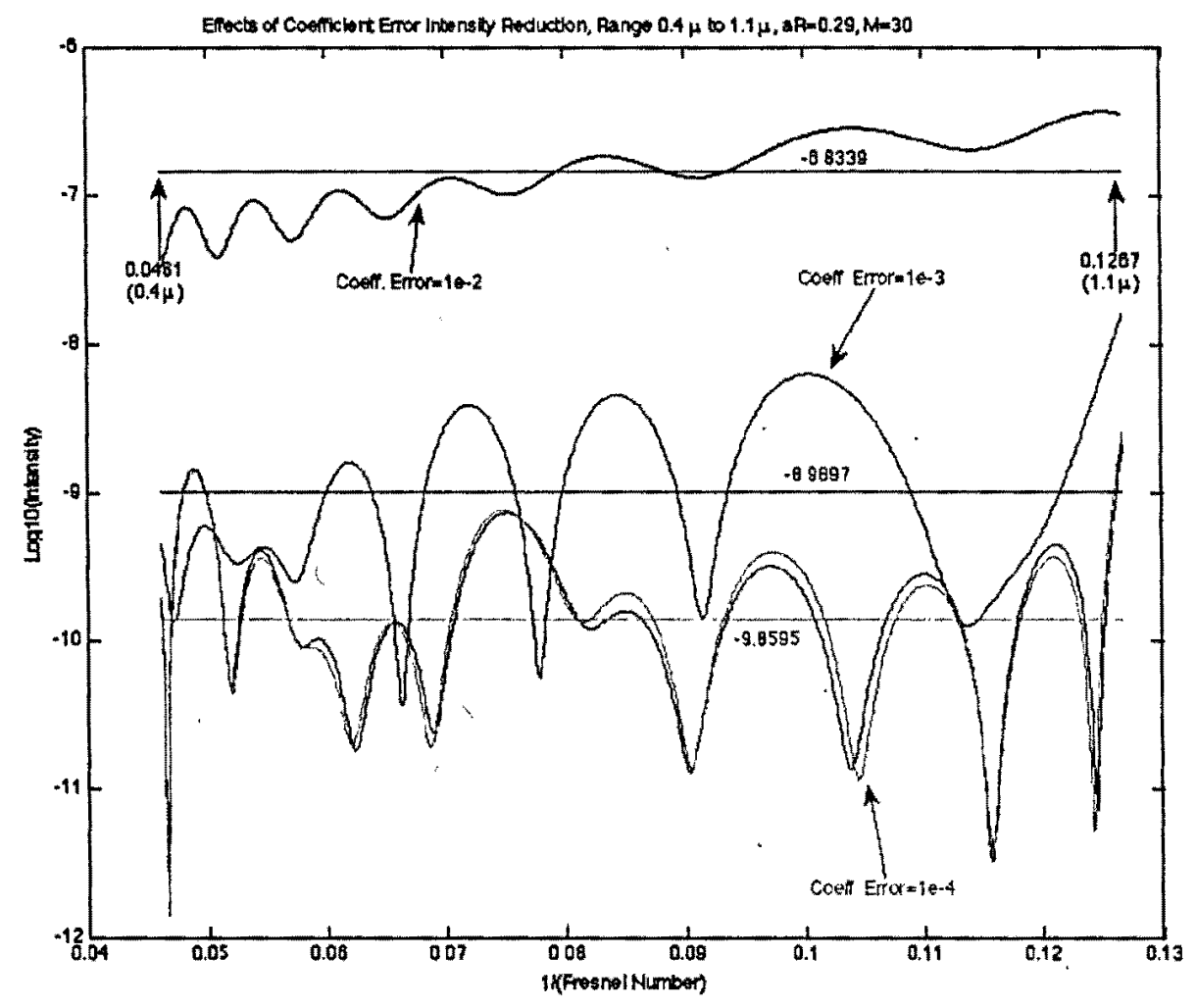

Figure 10. Sensitivity of scaled transparency function modeled based on perturbation of polynomial coefficients.

The intensity plots in this figure are based on scaled transparency polynomials of $30^{\text {th }}$ order. As the green plot show the coefficient perturbation in order of 1e-3 diminish the intensity from 9.8559 to 8.9897 .

For the special case of detecting Earth-like planets orbiting bright stars within the visible range (0.38-0.75 microns) requiring 10 orders of magnitude reduction in intensity demands transparency functions with polynomials of 40 degrees. Figure 11 shows an optimized the transparency function for this case normalized to the radius of the occulter disk of 25 meters. 


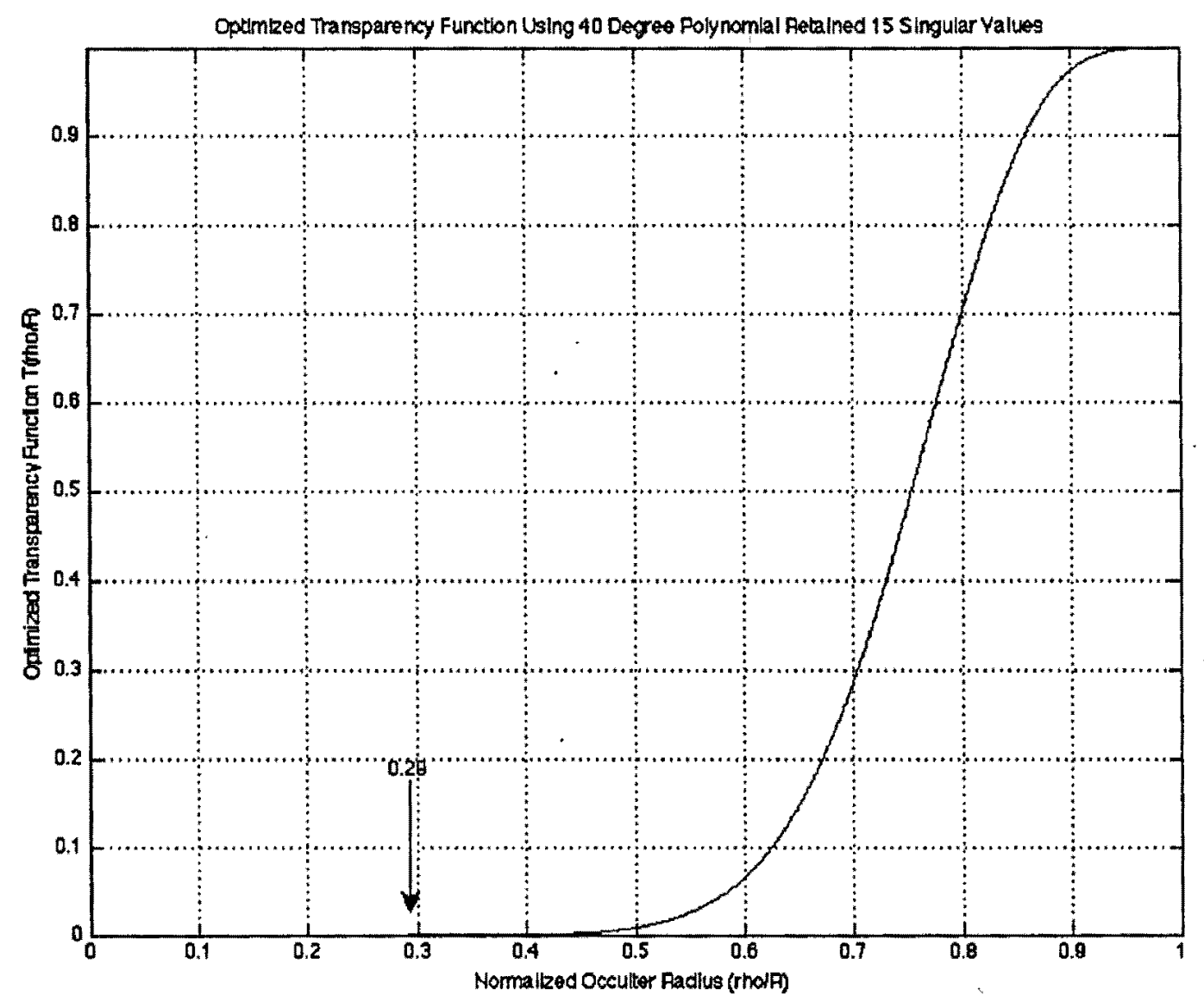

Figure 11. Transparency function of $\mathbf{4 0}$ degrees polynomlal optimized to the radius of occulter disk used in Exo-planet missions.

The intensity reduction along the central axis obtained from above function is plotted in Figure 12. In this case, the average intensity is around 11.4859 for a polychromatic source that meets or exceeds the requirements of detecting Exo-planets. 


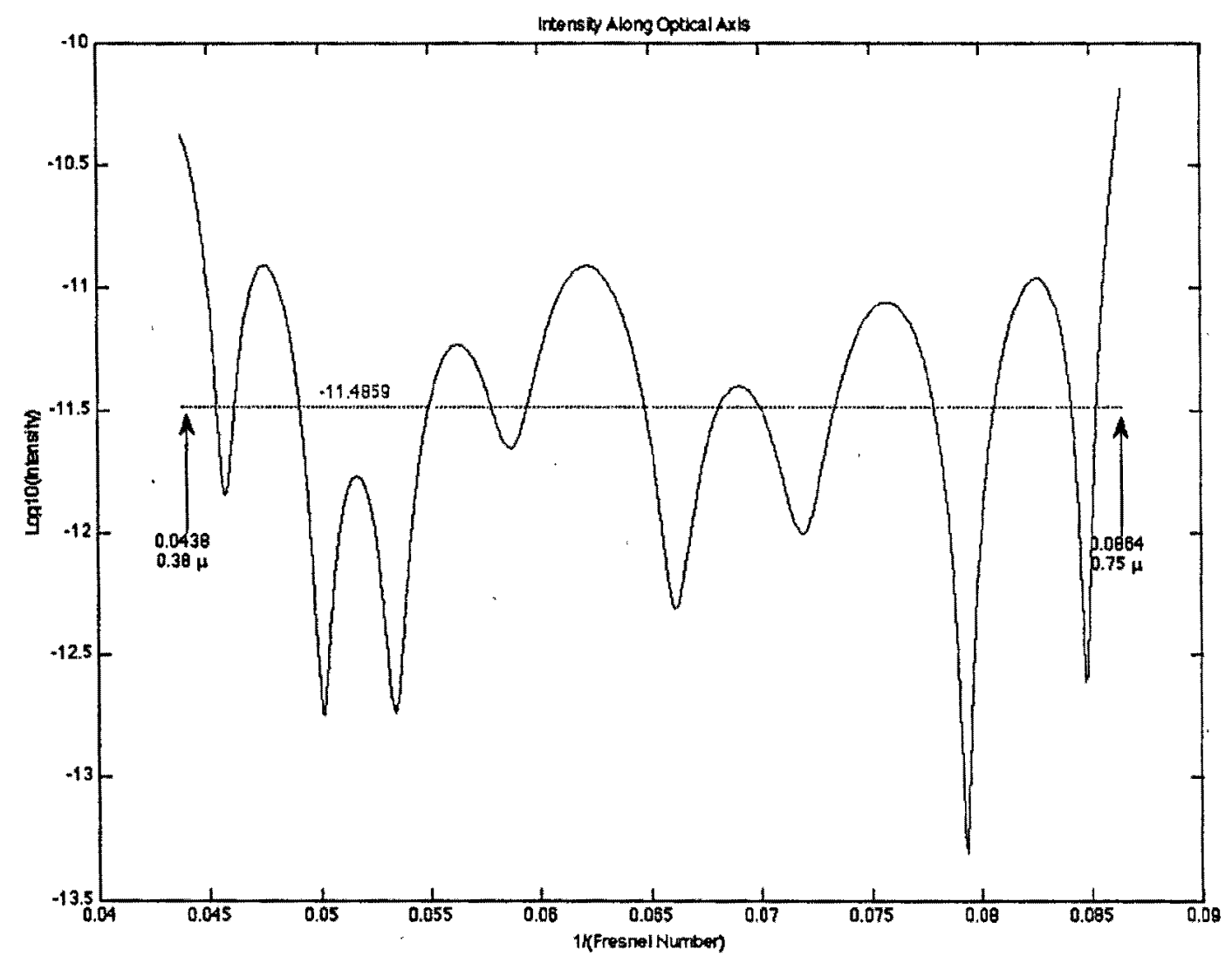

Figure 12. Intensity reduction along optical axis normalized to the Inverse of Fresnel Number for an optimized transparency with $\mathbf{4 0}$ degrees of polynomial retained 15 singular values for a range of 0.38 microns to 0.75 microns.

The transverse intensity at the location of telescope $72,000 \mathrm{~km}$ from the occulter disk at the location of telescope is shown in Figure 13. The radial intensity at the opening of telescope is maintained at 10 orders of magnitude for a 5-meter diameter of polychromatic range. 


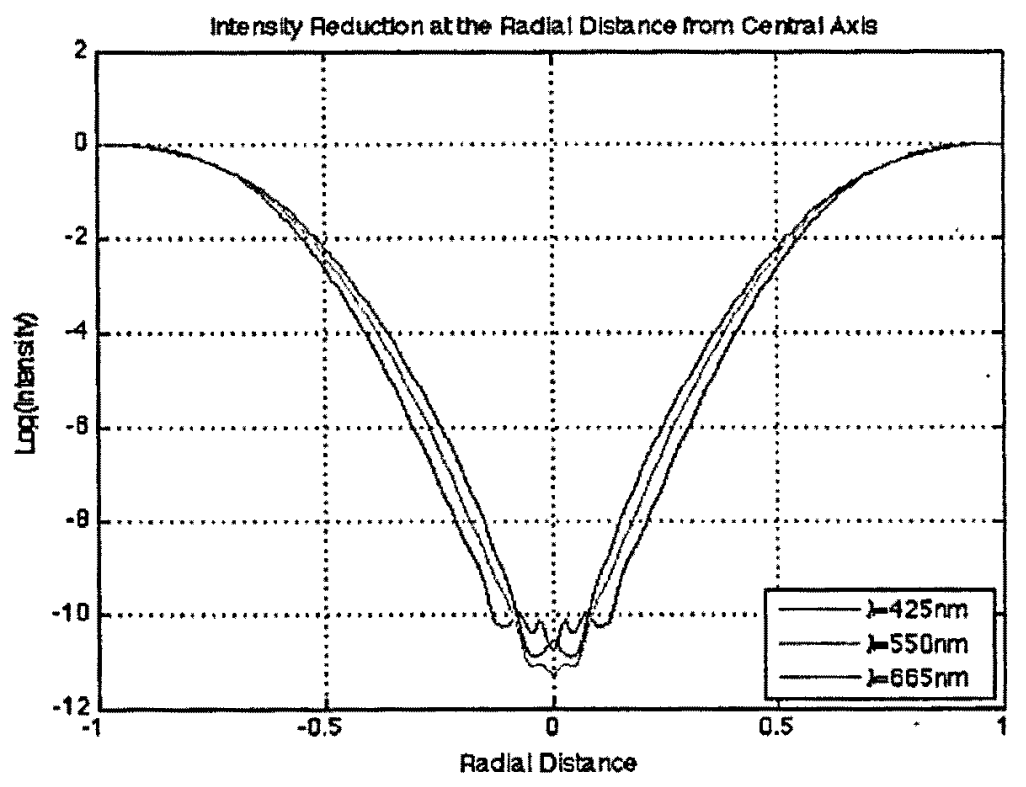

Figure 13. Transverse intensity at the entrance of telescope due to occultation by optimized circular disk

\section{Conclusion}

The study of Poisson Spot and methods of extinguishing this spot using simple circular occulter shows that the best way to reduce the intensity at this spot is the transparent occulters. These occulters are extremely sensitive to small deviations as small as $10^{-4}$ to the prescribed functional form of the taper. The average intensity reduction of 11 orders of magnitude could be obtained for astronomy missions such as Terrestrial Planet Finder with scaled optimized transparency function where the center of the disk is completely opaque. The performance of the petaled style occulters is certainly limited by the necessarily finite radius of the curvature at the petal tips. To achieve an intensity reduction approaching $10^{-8}$ would require the tip of the radii of curvature be on the order of 20 to 40 microns. Further, the reduction of the intensity along the central-axis of the occulter is independent of the number of the petals employed. 


\section{References}

1. C. Copi and G. D. Starkman, "The big occulting steerable satellite (BOSS)", ApJ 532, $581-592(2000)$

2. P. Nisenson, C. Papaliolios, "Detection of Earth-like planets using apodized telescopes", ApJ, 548, L201 (2001)

3. M. J. Kuchner and W. A. Traub, "A coronagraph with a band-limited mask for finding terrestrial planets", ApJ 579, 900 (2002)

4. J. C. Carson, D. W. Wilson, J. T. Trauger, "Coronagraphic masks for high-contrast nearinfrared imaging", Proc. SPIE, 5905, 59051D1-59051D-12 (2005)

5. W. Cash, "Detection of Earth-like planets around nearby stars using a petal-shaped occulter", Nature, 442, 51-53 (2006)

6. R. J. Vanderbei, E. Cady, and N. J. Kasdin, "Optical occulter design for finding extrasolar planets", ApJ, 665, 794-798 (2007) 